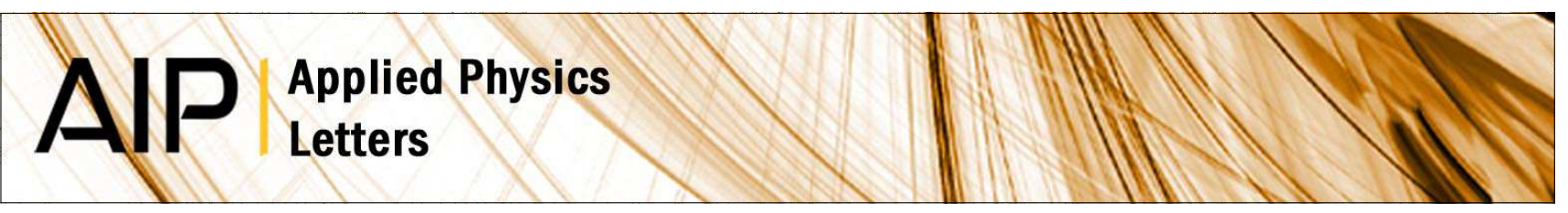

\title{
Optical pumping and reversal of hole spin in InAs/GaAs quantum dots
}

F. Fras, B. Eble, F. Bernardot, C. Testelin, M. Chamarro et al.

Citation: Appl. Phys. Lett. 100, 012104 (2012); doi: 10.1063/1.3673828

View online: http://dx.doi.org/10.1063/1.3673828

View Table of Contents: http://apl.aip.org/resource/1/APPLAB/v100/i1

Published by the American Institute of Physics.

Additional information on Appl. Phys. Lett.

Journal Homepage: http://apl.aip.org/

Journal Information: http://apl.aip.org/about/about_the_journal

Top downloads: http://apl.aip.org/features/most_downloaded

Information for Authors: http://apl.aip.org/authors

\section{ADVERTISEMENT}

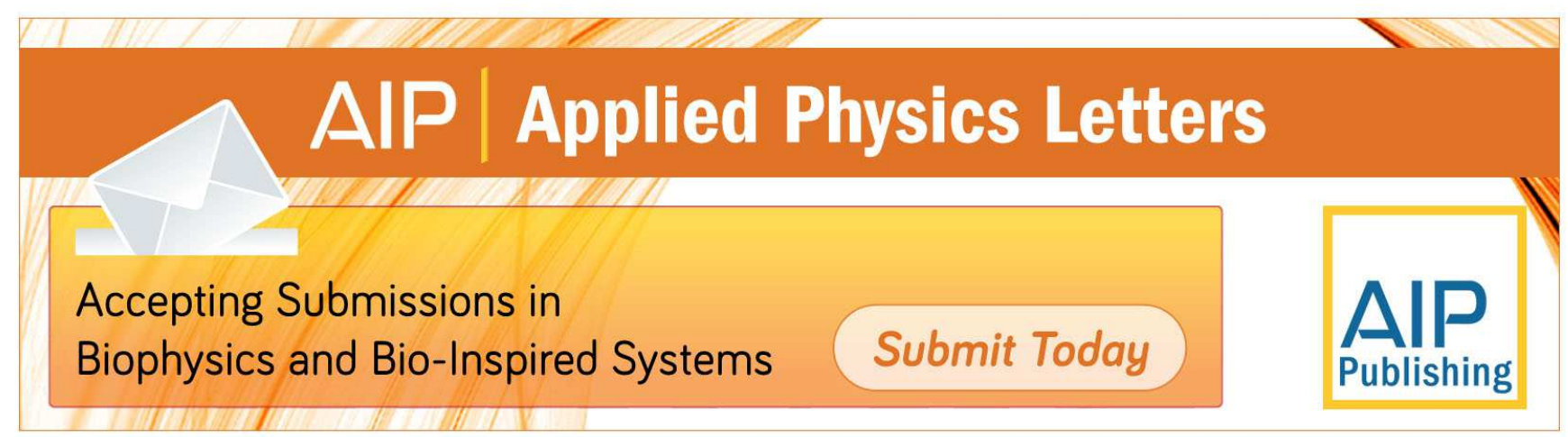




\title{
Optical pumping and reversal of hole spin in InAs/GaAs quantum dots
}

\author{
F. Fras, ${ }^{1}$ B. Eble, ${ }^{1}$ F. Bernardot, ${ }^{1}$ C. Testelin, ${ }^{1}$ M. Chamarro, ${ }^{1, a)}$ A. Miard,${ }^{2}$ and A. Lemaître ${ }^{2}$ \\ ${ }^{1}$ Institut des NanoSciences de Paris, UPMC Univ Paris 06, CNRS UMR 7588, 4 place Jussieu, \\ F-75252 Paris Cedex 05, France \\ ${ }^{2}$ Laboratoire de Photonique et Nanostructures, CNRS, Route de Nozay, F-91460 Marcoussis, France
}

(Received 4 November 2011; accepted 10 December 2011; published online 4 January 2012)

\begin{abstract}
We have obtained the optical pumping of hole spins, in p-doped InAs/GaAs quantum dots, via the generation of an intermediate trion state by a train of circularly polarized pulses. We show that we can optically control the orientation of the initialized hole spin, independently of the orientation of the intermediate trion state, by choosing the excitation energy of the circularly polarized light. This brings a supplementary degree of freedom for hole-spin manipulations in quantum dots. (C) 2012 American Institute of Physics. [doi:10.1063/1.3673828]
\end{abstract}

A single spin confined in a quantum dot (QD) is a potential candidate for applications in nanoscale spintronics and as a solid-state qubit. ${ }^{1-3}$ During the past years, the contact electron-nuclear spin interaction has been identified as the ultimate limit for these applications. Recently, several studies have been centred on hole spins since the p-type character of the valence-band Bloch functions results in a weaker hole-nuclear spin interaction and then in longer dephasing times. ${ }^{4-11}$ The initialization of carrier spins is a key process in the framework of information processing. In the last years, a special attention has been devoted to the all-optical approach which allows very high-speed spin manipulations, in addition to spin polarization and readout. Optical pumping, in particular, leads to the spin polarization and initialization via the absorption of circularly polarized photons. Hence, hole-spin initialization has been accomplished by an optical excitation, circularly polarized and resonant with the trion transition, ${ }^{4,7}$ or by the ionization of a spin-polarized photo-created electron-hole pair. ${ }^{12}$ In this letter, we report the hole-spin optical pumping by using an excitation tuned either at the ground-state trion transition or at higher-energy continuum states provided by the wetting layer (WL). For both excitations, the trion state is an intermediate state in the hole-spin polarization process. We show that we can optically control the orientation of the polarized hole spin, independently of the orientation of the intermediate trion state, by choosing the excitation energy of the circularly polarized light.

The investigated sample contains 30 planes of chemically p-doped InAs/GaAs QDs grown by molecular-beam epitaxy on a (001) GaAs substrate. More details about this sample are given in Ref. 7. The photoluminescence (PL) spectrum at $2 \mathrm{~K}$ is centred at $1.36 \mathrm{eV}$ and has a half width at half maximum of about $15 \mathrm{meV}$.

To probe the resident hole-spin polarization, we have measured the photo-induced circular dichroism (PCD). ${ }^{13} \mathrm{We}$ used two synchronized mode-locked Ti:sapphire lasers producing trains of 2-ps pulses with a $76-\mathrm{MHz}$ repetition rate. This two-laser-cavity scheme allows us to independently choose the pump and probe energies. The pump-beam polar-

\footnotetext{
${ }^{\text {a) }}$ Author to whom correspondence should be addressed. Electronic mail: maria.chamarro@insp.jussieu.fr.
}

ization is $\sigma^{+} / \sigma^{-}$modulated at $42 \mathrm{kHz}$ with a photo-elastic modulator, and the pump energy is tuned at different optical transitions of the sample. The probe beam is linearly polarized and tuned at $1.36 \mathrm{eV}$, the energy of the first optical transition of the optically selected ensemble of InAs/GaAs QDs. After transmission through the sample, the probe beam is analysed into its two circular components, and the difference in their intensities is measured with a balanced optical bridge. To improve the signal-to-noise ratio, a double lock-in amplifier analysis of the signal is performed, the pump and probe beams being modulated with a mechanical chopper at two different frequencies.

Considering pure heavy-hole (hh) states, the PCD signal has two contributions: (1) the population difference $\rho_{+3 / 2}-\rho_{-3 / 2}$ of the spin-polarized hh ground states $J_{z}= \pm 3 / 2\left(\rho_{ \pm 3 / 2}\right.$ being the $\pm 3 / 2$ hole state population $)$ and (2) the population difference $\rho_{+1 / 2}-\rho_{-1 / 2}$ of the spinpolarized trion states $S_{z}= \pm 1 / 2\left(\rho_{ \pm 1 / 2}\right.$ being the $\pm 1 / 2$ trion state population). Thus, the PCD signal satisfies $P C D \propto\left(\rho_{+3 / 2}-\rho_{-3 / 2}\right)-\left(\rho_{+1 / 2}-\rho_{-1 / 2}\right)$. As already demonstrated elsewhere, the optical pumping of the resident holes in p-doped QDs can be obtained by an optical pulsed excitation, circularly polarized and resonant with the trion transition. ${ }^{7,14}$ The inset of Fig. 1(a) shows the PCD signal obtained for a resonant excitation as function of pump-probe delay. We have tuned the two synchronized mode-locked Ti:sapphire lasers at the same energy, $1.36 \mathrm{eV}$. The presence of a non-zero PCD signal at pump-probe delays larger than the trion lifetime $\left(T_{R} \approx 800 \mathrm{ps}\right)$, and comparable to the repetition period $T_{L} \approx 13 \mathrm{~ns}$ of the pump pulses, is a signature of the optical pumping of the resident hole spins. Figure 2(a) shows the mechanism for the resonant optical pumping of the hole spins. The $\sigma^{+}$pump beam at normal incidence creates a hole with angular momentum $J_{z}=+3 / 2$ and an electron with spin $S_{z}=-1 / 2$. The efficient hyperfine interaction of the photo-created electron imposes a coherent coupling of both electron spin projections during the trion lifetime and leads, after emission of a $\sigma^{-}$photon, to a positive hole-spin polarization: $\rho_{+3 / 2}>\rho_{-3 / 2}$.

When the pump-beam energy is resonant with the WL optical transition, at $1.44 \mathrm{eV}$, the obtained PCD curve is clearly modified [see Fig. 1(a)]: a non-zero signal at negative pump-probe delays is still observed, but for this excitation 


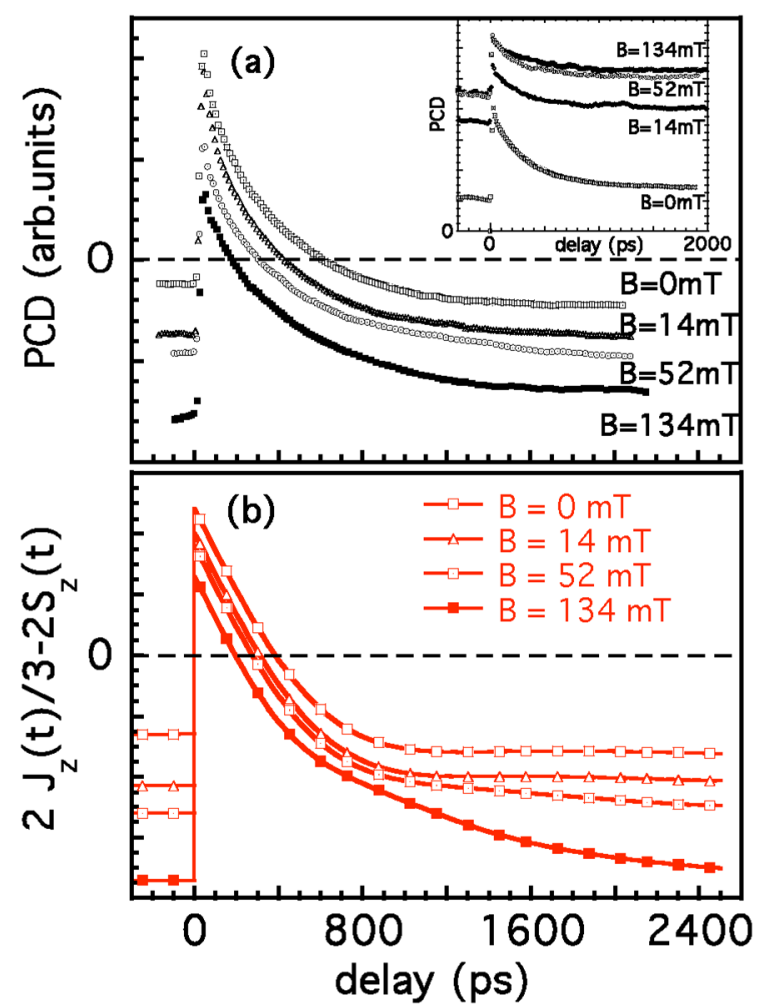

FIG. 1. (Color online) (a) PCD signals versus pump-probe delay obtained at $2 \mathrm{~K}$, under a non-resonant excitation (at $1.44 \mathrm{eV}$ ), and for different values of an applied longitudinal magnetic field. [Inset: PCD signals under a resonant excitation; the pump energy is tuned at $1.36 \mathrm{eV}$, the energy of the probe beam.] (b) Theoretical curves of PCD: $P C D(t) \propto 2 J_{z}(t) / 3-2 S_{z}(t)$, for a $\sigma^{+}$ excitation in the WL. We have used the same parameters as in Ref. 14, and $f=0.5, p_{e}=0.9$, and $p_{h}=0.1$.

energy, it becomes negative while the signal at short delays does not change its sign. As the PCD signal at negative delays writes $P C D\left(0^{-}\right) \propto \rho_{+3 / 2}-\rho_{-3 / 2}$, the sign modification of this long-living signal means that a reversal of the hole-spin polarization has been obtained, without reversal of the transient trion-spin polarization. A hole-spin reversal is also observed when the pump beam excites mixed states ${ }^{15}$ involving one QD-bound electron and one delocalized hole (e.g., at $1.39 \mathrm{eV}$ ).

Figure 2(b) shows a scheme of the non-resonant optical pumping of the hole spins, through a $\sigma^{+}$excitation of the WL transition. Previous works ${ }^{7,16}$ show that in p-doped QDs, the trion PL is co-polarized according to the laser helicity, which implies that the electron spins do not predominantly flip before recombination. One deduces that, following an optical excitation within the WL, the initial hole-spin orientation is lost due to the efficient spin relaxation process in the WL, whereas the electron-spin polarization partially remains after the capture and energy relaxation processes. ${ }^{16}$ After trion recombination (with emission of a $\sigma^{+}$photon), it is then possible to observe a hole-spin polarization with a sign opposite to the one obtained under resonant excitation: $\rho_{+3 / 2}<\rho_{-3 / 2}$.

In a previous work, we have developed a model of the interconnected dynamics of the photo-created electron spin and the resident hole spin, triggered through the optical excitation by a train of short pulses. This model is very useful to describe the temporal behaviour of the PCD when an applied (a) Resonant excitation

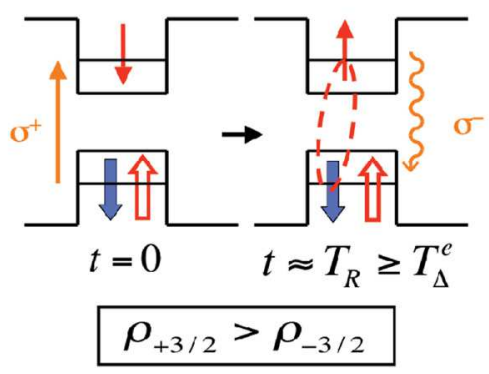

(b) Non-resonant excitation

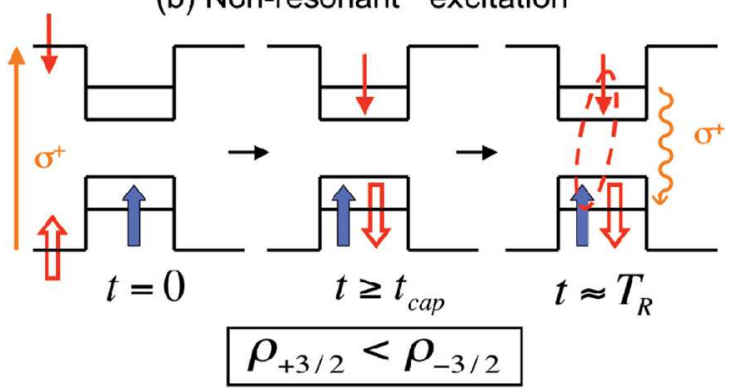

FIG. 2. (Color online) Mechanisms of optical pumping of the hole-spin polarization in p-doped InAs/GaAs QDs (a) under a $\sigma^{+}$excitation resonant with the trion transition and (b) under a non-resonant $\sigma^{+}$excitation in the WL. Arrow $\Downarrow$ (or $\Uparrow$ ) represents resident [full blue] or photo-created [hollow red] hole spin $J_{z}=-3 / 2$ (or $J_{z}=+3 / 2$ ); arrow $\downarrow$ (or $\uparrow$ ) represents the photo-created electron $\operatorname{spin} S_{z}=-1 / 2$ (or $\left.S_{z}=+1 / 2\right)$.

magnetic field screens the hyperfine interaction. ${ }^{14}$ Here, we have adapted this model to a non-resonant excitation. Basically, as for a resonant excitation, we assume that the dynamics of the electron spin, $\vec{S}$, and the one of the resident hole spin, $\vec{J}$, follow these two equations:

$$
\begin{gathered}
\frac{d \vec{S}(t)}{d t}=\frac{g_{e} \mu_{B}}{\hbar}\left(\vec{B}_{N}^{e}+\vec{B}\right) \times \vec{S}(t)-\frac{\vec{S}(t)}{T_{R}}, \\
\frac{d \vec{J}(t)}{d t}=\frac{g_{h} \mu_{B}}{\hbar}\left(\vec{B}_{N}^{h}+\vec{B}\right) \times \vec{J}(t)+3 \frac{(\vec{S} \cdot \vec{z}) \vec{z}}{T_{R}}-\frac{\vec{J}(t)}{T_{1}},
\end{gathered}
$$

where $\vec{z}$ is the unit vector along the growth direction of the sample, $\mu_{B}$ is the Bohr magneton, and $g_{e}$ and $g_{h}$ are the Landé factors of the electron and hole, respectively. $\vec{B}_{N}^{e}\left(\vec{B}_{N}^{h}\right)$ is the effective nuclear field induced by hyperfine coupling and acting on the electron (the hole); $\vec{B}=B \vec{z}$ is the applied magnetic field. $T_{R}$ is the trion lifetime, and $T_{1}$ is the long-scale relaxation time of the hole spin. ${ }^{17}$ A periodicity condition is also imposed: $\vec{J}\left(T_{L}^{-}\right)=\vec{J}\left(0^{-}\right)$, where $T_{L}$ is the period of the pulsed excitation and the superindex $(-)$ means before the pump pulse. For a resonant excitation, the $z$ component of the photocreated electron spin $S_{z}\left(0^{+}\right)$of the resident hole spin $\left.J_{z}\left(0^{+}\right)\right\}$, after a $\sigma^{+}$-polarized pump pulse with pulse area $\Theta$, is written as $S_{z}\left(0^{+}\right)=\sin ^{2}(\Theta / 2)\left[2 J_{z}\left(0^{-}\right) / 3-1\right] / 4\left\{J_{z}\left(0^{+}\right)\right.$ $\left.=\left[1-\sin ^{2}(\Theta / 2) / 2\right] J_{z}\left(0^{-}\right)+3 \sin ^{2}(\Theta / 2) / 4\right\}$. These last equations have to be changed under non-resonant excitation conditions, because, contrarily to the resonant case, all the QDs monitored by the probe beam energy are able to capture an electron-hole pair, independently of the spin of their resident hole. ${ }^{18}$

For a non-resonant excitation, the capture process by the QDs is considered very fast as compared to the radiative 
time of excitons in the WL. The kinetic equations describing the capture dynamics can be written as follows:

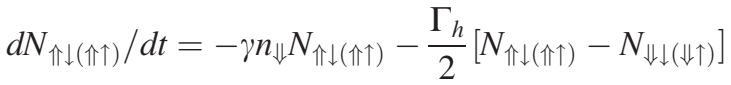

$$
\begin{aligned}
& -\frac{\Gamma_{e}}{2}\left[N_{\Uparrow \downarrow(\Uparrow \uparrow)}-N_{\Uparrow \uparrow(\Uparrow \downarrow)}\right], \\
& d N_{\Downarrow \uparrow(\Downarrow \downarrow)} / d t=-\gamma n_{\Uparrow} N_{\Downarrow \uparrow(\Downarrow \downarrow)}-\frac{\Gamma_{h}}{2}\left[N_{\Downarrow \uparrow(\Downarrow \downarrow)}-N_{\Uparrow \uparrow(\Uparrow \downarrow)}\right] \\
& -\frac{\Gamma_{e}}{2}\left[N_{\Downarrow \uparrow(\Downarrow \downarrow)}-N_{\Downarrow \downarrow(\Downarrow \uparrow)}\right], \\
& d n_{\Uparrow(\Downarrow)} / d t=-\gamma n_{\Uparrow(\Downarrow)}\left[N_{\Downarrow \downarrow(\Uparrow \downarrow)}+N_{\Downarrow \uparrow(\Uparrow \uparrow)}\right], \\
& d T_{\downarrow(\uparrow)} / d t=\gamma n_{\Uparrow} N_{\Downarrow \downarrow(\Downarrow \uparrow)}+\gamma n_{\Downarrow} N_{\Uparrow \downarrow(\Uparrow \uparrow)} .
\end{aligned}
$$

Equations (3) and (4) concern the four rate equations for the concentrations of all spin states of the bright $\left(N_{\Uparrow \downarrow}, N_{\Downarrow \uparrow}\right)$ and dark $\left(N_{\Uparrow \uparrow}, N_{\Downarrow \downarrow}\right)$ excitons. Equations (5) and (6) concern two rate equations for the concentrations $n_{\Uparrow}$ or $n_{\Downarrow}$ of the p-doped QDs with a resident spin-up or spin-down hole, and other two equations for the concentrations $T_{\uparrow}$ or $T_{\downarrow}$ of trions with a spin-up or spin-down electron. $\Gamma_{h}\left(\Gamma_{e}\right)$ denotes the hole-spin (electron-spin) relaxation rate in the WL. $\gamma$ is a phenomenological spin-independent cross section of capture by the QDs. Because of the capture terms, the Eqs. (3)-(6) are nonlinear. If the concentration $N_{0}$ of $\Uparrow \downarrow$ excitons created by the $\sigma^{+}$pump pulse is small as compared to the concentration $n_{0}$ of the studied p-doped QDs, it is possible, to first order in the capture fraction $f=N_{0} / n_{0}$, to keep $n_{\Uparrow(\Downarrow)} \approx n_{\Uparrow(\Downarrow)}\left(0^{-}\right)$constant in Eqs. (3) and (4). One then deals with linear equations, which can be analytically solved. Defining a capture rate $\Gamma=\gamma n_{0} / 2$ and assuming $\Gamma_{h} \gg \Gamma, \Gamma_{e},{ }^{19,20}$ one finds the following relations on the resident-hole-spin and trion-spin $z$ components, relating their values after excitonic capture with those before the $\sigma^{+}$pump pulse:

$$
\begin{gathered}
J_{z}\left(0^{+}\right)=\left[1-f\left(1-p_{h}\right)\right] J_{z}\left(0^{-}\right)+\frac{3}{2} f p_{h}, \\
S_{z}\left(0^{+}\right)=-\frac{1}{2} f p_{e},
\end{gathered}
$$

where $p_{e}\left(p_{h}\right)$ is the fraction of the electron-(hole-)spin polarization remaining after the capture process: $p_{e, h}=\left[1+\Gamma_{e, h} / \Gamma\right]^{-1}$. We can consider two limit cases: (1) if the hole depolarization is perfect in the $\mathrm{WL}, p_{h}=0$, and as expected, the hole-spin polarization at $t=0^{+}$is the fraction $1-f$ of the one existing before the pulse $(1-f$ is the fraction of the QDs which have not undergone capture) and (2) if the initial system is unpolarized, $J_{z}\left(0^{-}\right)=0$, and the hole $J_{z}\left(0^{+}\right)$and trion $S_{z}\left(0^{+}\right)$spin polarizations arise from the fraction $f$ of QDs which have undergone capture, taking into account the maintaining factors $p_{e, h}$.

For a non-resonant excitation, it is then possible to calculate $P C D(t) \propto 2 J_{z}(t) / 3-2 S_{z}(t)$ for different values of an external longitudinal magnetic field [see Fig. 1(b)]. The agreement with Fig. 1(a) is fairly good. Our simple model remarkably reproduces, for acceptable $f, p_{e}$, and $p_{h}$ parameters, all the experimental trends shown in Fig. 1(a): the calculated curves show a negative signal at negative delays and a positive signal at short-time delays after the pump pulse. Moreover, the PCD signal becomes more and more negative for increasing magnetic fields, as for the experimental data of Fig. 1(a). Nevertheless, slight discrepancies are observable: in absence of applied magnetic field, the calculated signal level at negative delays is too high, and at high magnetic field, the temporal signal behaviour is different from the experimental one.

Figure 3(a) shows the measured magnetic field dependence of the PCD signal at a negative delay, for a nonresonant excitation (at $1.44 \mathrm{eV}$ ). Two regimes are clearly identified: first, in the range $0-10 \mathrm{mT}$, a fast increase with increasing magnetic field and second, for $|\mathrm{B}|>10 \mathrm{mT}$, a slower magnetic field evolution. Figure 3(b) shows these two regimes in the calculated curve $P C D\left(0^{-}\right) \propto J_{z}\left(0^{-}\right)$. The low-magnetic-field regime corresponds to the screening of the hole hyperfine interaction, and the slower increase of the negative PCD signal in the range $\mathrm{B}=10-100 \mathrm{mT}$ is associated to the screening of the electron hyperfine interaction. ${ }^{7-9,14}$ In this latter range of magnetic field, the magnitude of hole-spin polarization increases more steeply in the experiment than what our simple model can reproduce. This may be due to an increase of the electron maintaining factor $p_{e}$ with magnetic field $\left(p_{e}\right.$ was kept constant in our model).

In conclusion, we have performed pump-probe experiments in p-doped InAs QDs leading to the optical pumping and read-out of the resident hole spins. For a resonant excitation, trions are directly photo-created in the QDs, and the hole-spin polarization arises from the electron hyperfine coupling in the trion state. For a non-resonant excitation, electron-hole pairs are photo-created in the WL, their capture by the QDs produces trions, and the hole-spin polarization comes from the preserved (lost) electron (hole) orientation in the WL. In both cases, an intermediate state, the trion, is created whose spin orientation is fixed by the helicity of the

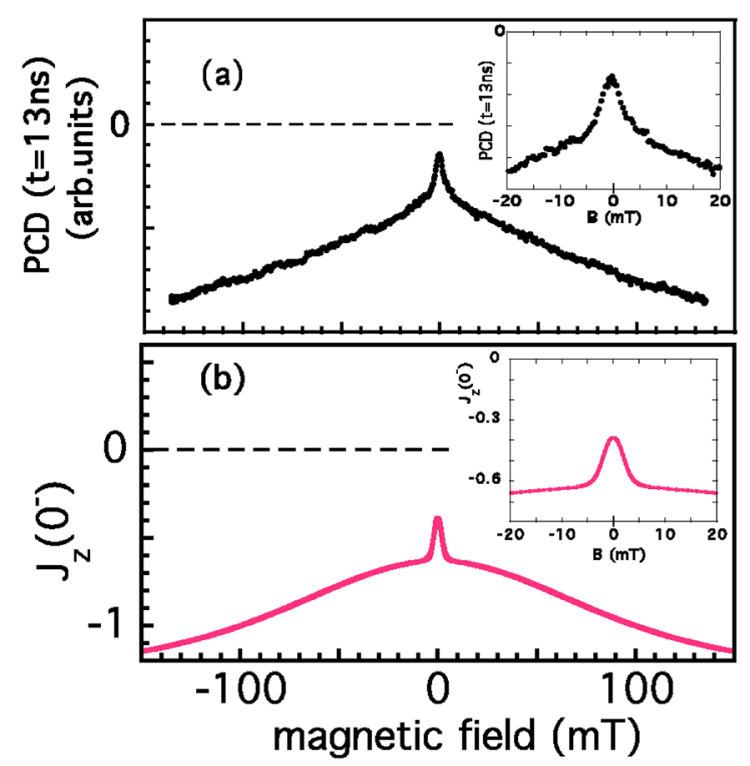

FIG. 3. (Color online) PCD signals at negative pump-probe delay as function of a longitudinal applied magnetic field, under a non-resonant $(1.44 \mathrm{eV})$ excitation: (a) experimental data; (b) calculated curve, where the used parameters are those mentioned in Fig. 1(b). [Insets: magnifications around zero magnetic field.] 
pump beam, independently of the final orientation of the resident hole, which is fixed by the excitation energy. Hence, for a non-resonant excitation, the optical pumping results in the inversion of the resident hole-spin polarization, with respect to the photo-created hole orientation. Finally, we have developed a model taking into account the coupled dynamics of the electron-hole-pair, trion, and hole spins; the calculations are in good agreement with the experiments performed in a longitudinal magnetic field.

We acknowledge support from the French ANR-P3N contract QUAMOS and region Île-de-France.

${ }^{1}$ D. Loss and D. P. DiVincenzo, Phys. Rev. A 57, 120 (1998).

${ }^{2}$ Semiconductor Spintronics and Quantum Computation, edited by D. D. Awschalom, D. Loss, and N. Samarth (Springer, New York, 2002).

${ }^{3}$ M. Chamarro, F. Bernardot, and C. Testelin, J. Phys.: Condens. Matter 19, 445007 (2007).

${ }^{4}$ B. D. Gerardot, D. Brunner, P. A. Dalgarno, P. Öhberg, S. Seidl, M. Kroner, K. Karrai, N. G. Stoltz, P. M. Petroff, and R. J. Warburton, Nature 451, 441 (2008).

${ }^{5}$ J. Fischer, W. A. Coish, D. V. Bulaev, and D. Loss, Phys. Rev. B 78, 155329 (2008).

${ }^{6}$ D. Brunner, B. D. Gerardot, P. A. Dalgarno, G. Wüst, K. Karrai, N. G. Stoltz, P. M. Petroff, and R. J. Warburton, Science 325, 70 (2009).

${ }^{7}$ B. Eble, C. Testelin, P. Desfonds, F. Bernardot, A. Balocchi, T. Amand, A. Miard, A. Lemaître, X. Marie, and M. Chamarro, Phys. Rev. Lett. 102, 146601 (2009).
${ }^{8}$ C. Testelin, F. Bernardot, B. Eble, and M. Chamarro, Phys. Rev. B 79, 195440 (2009).

${ }^{9}$ P. Desfonds, B. Eble, F. Fras, C. Testelin, F. Bernardot, M. Chamarro, B. Urbaszek, T. Amand, X. Marie, J.-M. Gérard, V. Thierry-Mieg, A. Miard, and A. Lemaître, Appl. Phys. Lett. 96, 172108 (2010).

${ }^{10}$ E. A. Chekhovich, A. B. Krysa, M. S. Skolnick, and A. I. Tartakovskii, Phys. Rev. Lett. 106, 027402 (2011).

${ }^{11}$ P. Fallahi, S. T. Yilmaz, and A. Imamoglu, Phys. Rev. Lett. 105, 257402 (2010).

${ }^{12}$ A. J. Ramsay, S. J. Boyle, R. S. Kolodka, J. B. B. Oliveira, J. Skiba-Szymanska, H. Y. Liu, M. Hopkinson, A. M. Fox, and M. S. Skolnick, Phys. Rev. Lett. 100, 197401 (2008).

${ }^{13}$ E. Aubry, C. Testelin, F. Bernardot, M. Chamarro, and A. Lemaître, Appl. Phys. Lett. 90, 242113 (2007).

${ }^{14}$ B. Eble, P. Desfonds, F. Fras, F. Bernardot, C. Testelin, M. Chamarro, A. Miard, and A. Lemaître, Phys. Rev. B 81, 045322 (2010).

${ }^{15}$ A. Vasanelli, R. Ferreira, and G. Bastard, Phys. Rev. Lett. 89, 216804 (2002); V. Popescu, G. Bester, and A. Zunger, Phys. Rev. B 80, 045327 (2009).

${ }^{16}$ P.-F. Braun, X. Marie, L. Lombez, B. Urbaszek, T. Amand, P. Renucci, V. K. Kalevich, K. V. Kavokin, O. Krebs, P. Voisin, and Y. Masumoto, Phys. Rev. Lett. 94, 116601 (2005), see also references therein.

${ }^{17}$ F. Fras, B. Eble, P. Desfonds, F. Bernardot, C. Testelin, M. Chamarro, A. Miard, and A. Lemaître, Phys. Rev. B 84, 125431 (2011).

${ }^{18} \mathrm{We}$ are neglecting any contribution of undoped QDs to the PCD signal, due to the linear symmetry of the neutral excitonic states (as discussed in Ref. 14).

${ }^{19}$ T. C. Damen, L. Viña, J. E. Cunningham, and J. Shah, Phys. Rev. Lett. 67, 3432 (1991).

${ }^{20}$ H. Kurtze, J. Seebeck, P. Gartner, D. R. Yakovlev, D. Reuter, A. D. Wieck, M. Bayer, and F. Jahnke, Phys. Rev. B 80, 235319 (2009). 